\title{
Detection and localization of a single binary trait locus in experimental populations
}

\author{
LAUREN M. MCINTYRE ${ }^{1,2,3 *}$, CYNTHIA J. COFFMAN ${ }^{3,4}$ AND R. W. DOERGE ${ }^{2,5}$ \\ ${ }^{1}$ Computational Genomics, Purdue University, West Lafayette, IN 47907, USA \\ ${ }^{2}$ Department of Agronomy, Purdue University, West Lafayette, IN 47907, USA \\ ${ }^{3}$ Duke University Medical Center, Division of Biometry, Durham, NC 27710, USA \\ ${ }^{4}$ Institute for Clinical and Epidemiological Research Biostatistics Unit, Durham VA Medical Center (152), Durham, NC 27705, USA \\ ${ }^{5}$ Department of Statistics, 1399 Mathematical Science Building, Purdue University, West Lafayette, IN 47907, USA
}

(Received 10 April 2000 and in revised form 19 December 2000)

\begin{abstract}
Summary
The advancements made in molecular technology coupled with statistical methodology have led to the successful detection and location of genomic regions (quantitative trait loci; QTL) associated with quantitative traits. Binary traits (e.g. susceptibility/resistance), while not quantitative in nature, are equally important for the purpose of detecting and locating significant associations with genomic regions. Existing interval regression methods used in binary trait analysis are adapted from quantitative trait analysis and the tests for regression coefficients are tests of effect, not detection. Additionally, estimates of recombination that fail to take into account varying penetrance perform poorly when penetrance is incomplete. In this work a complete probability model for binary trait data is developed allowing for unbiased estimation of both penetrance and recombination between a genetic marker locus and a binary trait locus for backcross and $\mathrm{F}_{2}$ experimental designs. The regression model is reparameterized allowing for tests of detection. Extensive simulations were conducted to assess the performance of estimation and testing in the proposed parameterization. The proposed parameterization was compared with interval regression via simulation. The results indicate that our parameterization shows equivalent estimation capabilities, requires less computational effort and works well with only a single marker.
\end{abstract}

\section{Introduction}

Statistical methods for mapping continuous traits have advanced from methods that consider a single marker and single trait (Wright, 1952; Mérat, 1968; Hammond \& James, 1970; O'Donald, 1971; Fain, 1978; Weller, 1986; Beckman \& Soller, 1988; Luo \& Kearsey, 1989; Luo \& Woolliams, 1993) to methods which map multiple quantitative trait loci (QTL) using information from many markers (Jansen, 1992, 1993; Zeng, 1993, 1994). These likelihood interval mapping methods have been extended to handle epistatic interactions between genes (Kao, 1995). Churchill \& Doerge (1994) and Doerge \& Churchill (1996) have introduced permutation testing as a mechanism for dealing with violations of assumptions.

\footnotetext{
* Corresponding author. Department of Agronomy, 1150 Lilly Hall of Science, Purdue University, West Lafayette, IN 47907 , USA. Tel: + 1 (765) 494 4773. Fax: + 1 (765) 496 2926. e-mail: lmcintyre@purdue.edu
}

Haley \& Knott (1992) and Martinez \& Curnow (1992) proposed regression-based methods that are easier to implement and require less computational effort than the likelihood interval mapping methods and have been shown to be comparable to the likelihood methods (Xu, 1996). For comprehensive reviews of these statistical methods, as well as an overview of the tissues involved in searching for genes, see Doerge et al. (1997), Elston (1998) and Olson et al. (1999).

Binary traits (e.g. susceptibility/resistance), while not quantitative in nature, are equally important for the purpose of detecting and locating significant associations with genomic regions. Much of the complication in binary traits comes from their seemingly simple descriptions (e.g., presence or absence), when in fact their underlying biological model may be more complicated. One approach to the analysis of binary trait data is to consider the trait as a genetic marker (Paterson, 1998), and then map the trait using genetic mapping methodology. Similarly, 
simple $\chi^{2}$ tests (e.g. Wilcox, 1995) using $2 \times 2$ associations have been used to test for associations between a single marker and a binary trait. While both these approaches provide information on the association of a binary trait locus (BTL) near previously mapped genetic markers, they do not estimate the genetic distance between the marker and BTL (recombination) and thus are unable to estimate the proportion of individuals for an underlying genotype that express the trait (penetrance).

Recombination for BTL has been estimated by adapting QTL analysis. Hacket \& Weller (1995) and $\mathrm{Xu} \&$ Atchley (1996) used a logistic regression approach applied to ordinal or binary trait data. Visscher et al. (1996) used a generalized linear model, and Kruglyak \& Lander (1995) proposed a nonparametric approach based on a generalization of the Wilcoxon rank test. Rebaï (1997) compared the methods proposed by Hackett \& Weller (1995) and Kruglyak \& Lander (1995) with the standard linear regression interval mapping methods (Haley \& Knott, 1992) for analysing BTL and reported that the linear regression approach was robust against non-normality and that the loss of power was not significant. Visscher et al. (1996) reported similar results in their comparison of generalized linear models and linear regression in their analysis of BTL. The linear and logistic approaches gave similar results in terms of location (recombination) and power for effect, demonstrating that the estimation of recombination between binary trait loci and markers is possible using the QTL (continuous) framework. Xu (1996) compared the performance of regression and maximum likelihood approaches and found that regressionbased approaches combined with permutation testing work well. In addition to this work, much has been done in human genetics, where looking at the association of multiple factors with a single binary outcome (disease status) is commonplace. Particularly relevant to the model we propose is an approach proposed by Thompson (1998) where the use of segregation indicators is described.

The existing models for binary traits in experimental populations define the hypothesis tests in terms of the effect. In addition, much of the prior work on BTL focuses on modelling an underlying threshold distribution (Xu \& Atchley, 1996; Xu, 1996). The threshold model is an important quantitative genetic model; however, the underlying threshold distribution is unobserved. What is observed is the cumulative probability of the distribution from the threshold point to the limit of the distribution function, or the observed proportion of individuals with the trait. Many different types of threshold models can give the same value for the threshold, and in many cases, the appropriate underlying threshold model is unknown. Ideally, a complete methodological framework for binary traits that allows detection of BTL and estimation of both the recombination and the penetrance between the BTL and the marker locus is needed. This methodology should be expandable, easily interpretable, easily implemented and accuracy of estimates should not require estimation of the threshold model.

In this work, we develop a probability model for a binary trait locus that is based on classical genetic theory. Implementation of the model using segregation indicator variables (Thompson, 1998) is straightforward, and combined with regression techniques can be used to detect associations between the marker and the BTL even when penetrance is incomplete. The standard tests of the regression coefficients are easily interpreted using the probability model regardless of the type of regression (linear or logistic) performed. The power of the tests in both the linear and logistic settings is examined using simulations and reported for the backcross and $F_{2}$ experimental designs. The addition of a second flanking marker is considered and estimates of recombination and penetrance are developed in both single and flanking marker cases. Simulations were performed for the purpose of evaluating the performance of these estimators and comparing them to those from interval regression.

\section{Methods}

(i) A probability model

Using notation established by Doerge et al. (1997), genetic markers will be represented by $\mathbf{M}$ and $\mathbf{N}$ and binary trait loci (BTLs) denoted by $\mathbf{Q}$. In the backcross and $\mathrm{F}_{2}$ designs, for diploid individuals (Fig. 1), there are only two possible alleles for each marker and/or BTL, and they will be denoted by $M_{1}, M_{2}$, and $Q_{1}, Q_{2}$. Recombination, $r_{M Q}$, is the probability that an observable exchange of genetic material occurred between the BTL $\left(Q_{1}\right.$ or $\left.Q_{2}\right)$ and the marker $\left(M_{1}\right.$ or $\left.M_{2}\right)$. The amount of recombination is a measure of association between the marker and the BTL. When $r_{M Q}=0 \cdot 50$, there is no association between the marker and the BTL.

In the backcross design, for each individual there are two possible marker types (MT), $M_{1} / M_{1}$ and $M_{1} / M_{2}$, and two BTL genotypes (GT), $Q_{1} / Q_{1}$ and $Q_{1} / Q_{2}$, giving a total of four possible combinations of marker type and genotype. The number of marker type and genotype combinations will be denoted by $c$ from this point forward. In the $F_{2}$, there are three marker types and three genotypes, giving a total of nine possible combinations of marker type and genotype $(c=9)$. The initiating parents are assumed to be homozygous inbred lines differing in the binary trait of interest, meaning that each distribution of the trait for Parent ${ }_{1}$ and Parent ${ }_{2}$ is a different binomial distribution (see Fig. 1 ) such that $p_{1} \neq p_{2}$. In addition, 
Table 1. Joint and conditional probability distributions for a backcross experimental population

\begin{tabular}{|c|c|c|c|c|c|c|}
\hline MT & $P(M T)$ & GT & $P(G T \mid M T)$ & $P(Y \mid G T)$ & $P(Y, G T \mid M T)$ & $P(Y, G T, M T)$ \\
\hline \multirow{2}{*}{$M_{1} / M_{1}$} & & $Q_{1} / Q_{1}$ & $\left(1-r_{M Q}\right)$ & $p_{1}$ & $\left(1-r_{M Q}\right) p_{1}$ & $\left(1-r_{M Q}\right) \frac{p_{1}}{2}$ \\
\hline & & $Q_{1} / Q_{2}$ & $r_{M Q}$ & $p_{3}$ & $r_{M Q} p_{3}$ & $r_{M Q} \frac{p_{3}}{2}$ \\
\hline \multirow[t]{2}{*}{$M_{1} / M_{2}$} & & $Q_{1} / Q_{1}$ & & $p_{1}$ & $r_{M Q} p_{1}$ & $r_{M Q} \frac{p_{1}}{2}$ \\
\hline & & $Q_{1} / Q_{2}$ & $\left(1-r_{M Q}\right)$ & $p_{3}$ & $\left(1-r_{M Q}\right) p_{3}$ & $\left(1-r_{M Q}\right) \frac{p_{3}}{2}$ \\
\hline
\end{tabular}

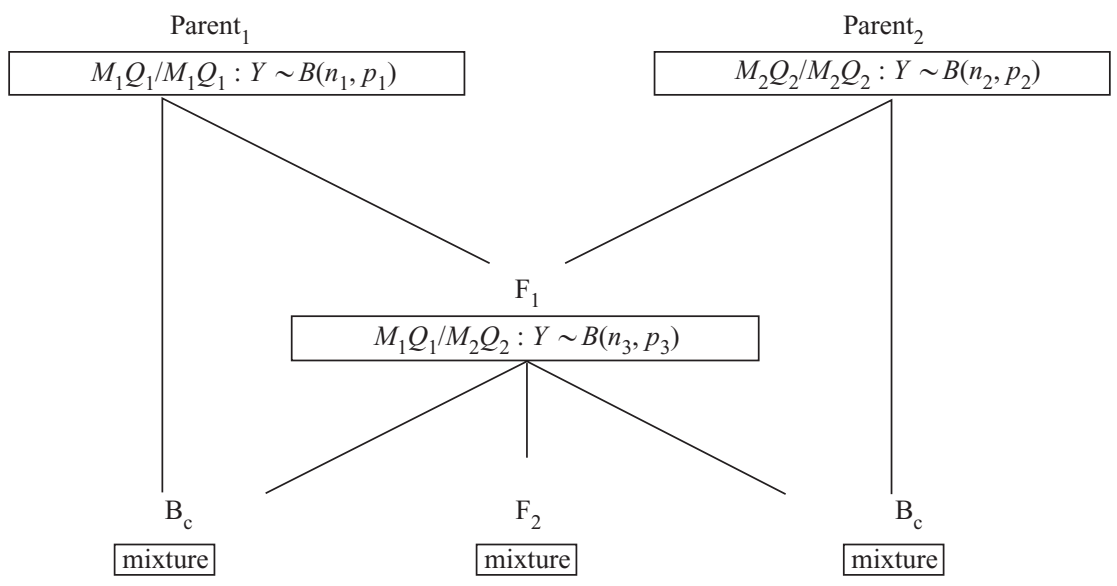

Fig. 1. Trait distributions for backcross and $\mathrm{F}_{2}$ design.

for clarity of further discussion and without loss of generality, we assume $p_{1}<p_{2}$. The $\mathrm{F}_{1}$ is assumed to be distributed binomially with $p_{3}$.

The binomial probabilities $p_{1}, p_{2}$ and $p_{3}$ shown in Fig. 1 represent the probability that a binary trait is present given a specific BTL genotype (GT), or the penetrance of the trait for the specific genotypes $Q_{1} / Q_{1}, Q_{1} / Q_{2}$ and $Q_{2} / Q_{2}$, respectively. A dominant model is the special case where $p_{2}=p_{3}$, and the recessive model is the special case where $p_{1}=p_{3}$. Other genetic models can be expressed as combinations of the $p_{i}$ (i.e. the midparent model is $p_{3}=\left(p_{1}+p_{2}\right) / 2$. In this parameterization, the dominant and recessive models are mathematical mirrors of each other.

The joint probability of the genotypes, marker types and the trait for specific experimental designs can be expressed in terms of $r_{M Q}, p_{1}, p_{2}$ and $p_{3}$. For the joint and conditional probability distributions of the backcross and the $\mathrm{F}_{2}$ experimental designs see Tables 1 and 2.

\section{(ii) Expected values}

The joint probability of $Y, G T, M T$ can be written as follows:

$$
\begin{aligned}
P(Y, G T, M T) & =P(Y \mid G T, M T) P(G T, M T) \\
& =P(Y \mid G T) P(G T, M T) \\
& =P(Y \mid G T) P(G T \mid M T) P(M T)
\end{aligned}
$$

where $P(Y \mid G T, M T)=P(Y \mid G T)=p_{i}$.
Given the joint probability of $Y, G T, M T$ the expected values $(E(Y))$ for individuals in a backcross or $\mathrm{F}_{2}$ population are as follows.

For the single marker, single BTL backcross model, where the $F_{1}$ is crossed with Parent $t_{1}$,

$$
\begin{aligned}
E(Y)= & Y \sum_{c} P(Y, G T, M T) \\
= & 1\left[\left(1-r_{M Q}\right) \frac{p_{1}}{2}+r_{M Q} \frac{p_{3}}{2}+r_{M Q} \frac{p_{1}}{2}\right. \\
& \left.+\left(1-r_{M Q}\right) \frac{p_{3}}{2}\right] \\
& +0\left[1-\left(1-r_{M Q}\right) \frac{p_{1}}{2}+1-r_{M Q} \frac{p_{2}}{2}+1-r_{M Q} \frac{p_{1}}{2}\right. \\
& \left.+1-\left(1-r_{M Q}\right) \frac{p_{3}}{2}\right] \\
= & \frac{p_{1}}{2}+\frac{p_{3}}{2},
\end{aligned}
$$

and similarly for the reciprocal backcross the $E(Y)=\frac{p_{2}}{2}+\frac{p_{3}}{2}$.

In the $\mathrm{F}_{2}$ design,

$$
\begin{aligned}
E(Y) & =Y \sum_{c} P(Y, G T, M T) \\
& =\frac{p_{1}}{4}+\frac{p_{3}}{2}+\frac{p_{2}}{4} .
\end{aligned}
$$

If Parent ${ }_{1}$ and Parent ${ }_{2}$ are extreme cases, such that $p_{1}=0$ and $p_{2}=1$, then in the dominant model 
Table 2. Joint and conditional probability distributions for an $\mathrm{F}_{2}$ experimental population

\begin{tabular}{lllllll}
\hline \hline MT & $P(M T)$ & $\mathrm{GT}$ & $P(G T \mid M T)$ & $P(Y \mid G T)$ & $P(Y, G T \mid M T)$ & $P(Y, G T, M T)$ \\
\hline$M_{1} / M_{1}$ & $\frac{1}{4}$ & $Q_{1} / Q_{1}$ & $\left(1-r_{M Q}\right)^{2}$ & $p_{1}$ & $\left(1-r_{M Q}\right)^{2} p_{1}$ & $\left(1-r_{M Q}\right)^{2} \frac{p_{1}}{4}$ \\
& & $Q_{1} / Q_{2}$ & $2\left(r_{M Q}\left(1-r_{M Q}\right)\right)$ & $p_{3}$ & $2\left(r_{M Q}\left(1-r_{M Q}\right) p_{3}\right)$ & $2\left(r_{M Q}\left(1-r_{M Q}\right) \frac{p_{3}}{4}\right)$ \\
& & $Q_{2} / Q_{2}$ & $r_{M Q}^{2}$ & $p_{2}$ & $r_{M Q}^{2} p_{2}$ & $r_{M Q}^{2} \frac{p_{2}}{4}$ \\
$M_{1} / M_{2}$ & $\frac{1}{2}$ & $Q_{1} / Q_{1}$ & $r_{M Q}\left(1-r_{M Q}\right)$ & $p_{1}$ & $r_{M Q}\left(1-r_{M Q}\right) p_{1}$ & $r_{M Q}\left(1-r_{M Q}\right) \frac{p_{1}}{2}$ \\
& & $Q_{1} / Q_{2}$ & $\left(1-r_{M Q}\right)^{2}$ & $p_{3}$ & $\left(1-r_{M Q}\right)^{2} p_{3}$ & $\left(1-r_{M Q}\right)^{2} \frac{p_{3}}{2}$ \\
& & $Q_{2} / Q_{1}$ & $r_{M Q}^{2}$ & $p_{3}$ & $r_{M Q}^{2} p_{3}$ & $r_{M Q}^{2} \frac{p_{3}}{2}$ \\
& & $Q_{2} / Q_{2}$ & $r_{M Q}\left(1-r_{M Q}\right)$ & $p_{2}$ & $r_{M Q}\left(1-r_{M Q}\right) p_{2}$ & $r_{M Q}\left(1-r_{M Q}\right) \frac{p_{2}}{2}$ \\
$M_{2} / M_{2}$ & $\frac{1}{4}$ & $Q_{1} / Q_{1}$ & $r_{M Q}^{2}$ & $p_{1}$ & $r_{M Q}^{2} p_{1}$ & $r_{M Q}^{2} \frac{p_{1}}{4}$ \\
& & $Q_{1} / Q_{2}$ & $2\left(r_{M Q}\left(1-r_{M Q}\right)\right)$ & $p_{3}$ & $2\left(r_{M Q}\left(1-r_{M Q}\right)\right) p_{3}$ & $2\left(r_{M Q}\left(1-r_{M Q}\right) \frac{p_{3}}{4}\right)$ \\
& & $Q_{2} / Q_{2}$ & $\left(1-r_{M Q}\right)^{2}$ & $p_{2}$ & $\left(1-r_{M Q}\right)^{2} p_{2}$ & $\left(1-r_{M Q}\right)^{2} \frac{p_{2}}{4}$ \\
\hline \hline
\end{tabular}

$\left(p_{2}=p_{3}\right), E(Y)=\frac{3}{4}$ and in the recessive model $\left(p_{1}=p_{3}\right), E(Y)=\frac{1}{4}$.

\section{(iii) Regression models}

Now that the probability model for BTL has been described in terms of its parameters $\left(r_{M Q}, p_{1}, p_{2}\right.$ and $p_{3}$ ), it can be combined with regression techniques to detect associations between a marker and the BTL. In this framework, the test for marker and BTL association is a test of the null hypotheses, $r_{M Q}=0 \cdot 50$. As previous work has focused on both linear and logistic regression, in this work we examined the interpretation of the tests of the regression coefficients in both a linear and logistic regression model using the probability model developed above (Tables 1,2 ).

\section{(a) Linear model}

The linear regression model parameterized for a backcross, single BTL, single marker model is written as:

$Y_{i}=\beta_{0}+\beta_{1} X_{i}+\epsilon_{i} ; \quad i=1, \ldots, n$,

where

$Y_{i}= \begin{cases}1, & \text { Trait }=1 \\ 0, & \text { otherwise }\end{cases}$

$X_{i}= \begin{cases}1, & \text { if } \mathrm{MT}=M_{1} / M_{2} \\ 0, & \text { if } \mathrm{MT}=M_{1} / M_{1} .\end{cases}$

This representation using the $X_{i}$ as indicator functions allows the parameters $\beta_{0}$ and $\beta_{1}$ in the regression model to be expressed as

$\beta_{0}=\mu_{M_{1} / M_{1}}$

$\beta_{1}=\mu_{M_{1} / M_{2}}-\mu_{M_{1} / M_{1}}$.

However, as with any linear model the error terms are assumed to be independent and normally distributed with mean zero and constant variance $\sigma^{2}$. While this does not affect the estimation of the regression coefficients (Searle, 1997), it can affect the distribution of the test statistic for the test $\beta_{1}=0$. Therefore, the distribution of the test statistic and the power of the test for the regression parameters must be carefully examined. In this paper, all $P$ values were determined using permutation theory (Churchill \& Doerge, 1994; Doerge \& Churchill, 1996).

Using the conditional probabilities given in Table 1, the difference between marker class means is expressed as a function of $r_{M Q}, p_{1}$ and $p_{3}$, where

$\mu_{M_{1} / M_{2}}-\mu_{M_{1} / M_{1}}=\left(1-2 r_{M Q}\right)\left(p_{3}-p_{1}\right)$.

In this case, the test of the regression coefficient $\beta_{1}=0$ is a test of $r_{M Q}=0.50$ and $p_{1}=p_{3}$. Assuming that $p_{1} \neq p_{3}$, this is a direct test of $r_{M Q}=0.50$.

Similarly, in an $\mathrm{F}_{2}$ population the linear regression model is written as:

$Y_{i}=\beta_{0}+\beta_{1} X_{1 i}+\beta_{2} X_{2 i}+\epsilon_{i} ; \quad i=1, \ldots, n$

where

$Y_{i}= \begin{cases}1, & \text { Trait }=1 \\ 0, & \text { otherwise }\end{cases}$

$X_{1 i}= \begin{cases}1, & \text { if } \mathrm{MT}=M_{1} / M_{2} \\ 0, & \text { otherwise }\end{cases}$

$X_{2 i}= \begin{cases}1, & \text { if MT }=M_{2} / M_{2} \\ 0, & \text { otherwise }\end{cases}$

Using the $X_{i}$ as specific indicator functions allows the regression the parameters $\beta_{0}, \beta_{1}$ and $\beta_{2}$ to be expressed as

$\beta_{0}=\mu_{M_{1} / M_{1}}$

$\beta_{1}=\mu_{M_{1} / M_{2}}-\mu_{M_{1} / M_{1}}$,

$\beta_{2}=\mu_{M_{2} / M_{2}}-\mu_{M_{1} / M_{1}}$

As with the backcross, the conditional probabilities given in Table 2 for the $F_{2}$ are used to express the 
Table 3. Estimates of $r_{M Q}$ and $p_{3}$ for single marker backcross and $\mathrm{F}_{2}$ populations

\begin{tabular}{lll}
\hline \hline & $\hat{r}_{M Q}$ & $\hat{p}_{3}$ \\
\hline Backcross & $\frac{p_{1}-\mu_{M_{1} / M_{1}}}{2 p_{1}-\mu_{M_{1} / M_{2}}-\mu_{M_{1} / M_{1}}}$ & $\mu_{M_{1} / M_{2}}+\mu_{M_{1} / M_{1}}-p_{1}$ \\
$\mathrm{~F}_{2}$ & $\frac{1}{2} \frac{p_{1}+\mu_{M_{2} / M_{2}}-\mu_{M_{1} / M_{1}}-p_{2}}{p_{1}-p_{2}}$ & $\frac{1}{2} \mu_{M_{2} / M_{2}}+\mu_{M_{1} / M_{2}}+\frac{1}{2} \mu_{\mathrm{M}_{1} / M_{1}}-\frac{1}{2} p_{1}-\frac{1}{2} p_{2}$ \\
\hline \hline
\end{tabular}

Table 4. Expected trait distributions for binary traits in a backcross with two markers for linkage map MQN

\begin{tabular}{lllllll}
\hline \hline MT & $P(M T)$ & GT & $P(G T \mid M T)$ & $P(Y \mid G T)$ & $P(Y, G T \mid M T)$ & $P(Y, G T, M T)$ \\
\hline$M_{1} N_{1} / M_{1} N_{1}$ & $\frac{1}{2}\left(1-r_{M N}\right)$ & $Q_{1} / Q_{1}$ & $\frac{\left(1-r_{M Q}\right)\left(1-r_{N Q}\right)}{1-r_{M N}}$ & $p_{1}$ & $\frac{\left(1-r_{M Q}\right)\left(1-r_{N Q}\right)}{1-r_{M N}} p_{1}$ & $\frac{1}{2}\left(1-r_{M Q}\right)\left(1-r_{N Q}\right) p_{1}$ \\
& & $Q_{1} / Q_{2}$ & $\frac{r_{M Q} r_{M Q}}{1-r_{M N}}$ & $p_{3}$ & $\frac{r_{M Q} r_{M Q}}{1-r_{M N}} p_{3}$ & $\frac{1}{2} r_{M Q} r_{N Q} p_{3}$ \\
$M_{1} N_{1} / M_{1} N_{2}$ & \multirow{2}{*}{$r_{M N}$} & $Q_{1} / Q_{1}$ & $\frac{\left(1-r_{M Q}\right)\left(r_{N Q}\right)}{r_{M N}}$ & $p_{1}$ & $\frac{\left(1-r_{M Q}\right)\left(r_{N Q}\right)}{r_{M N}} p_{1}$ & $\frac{1}{2}\left(1-r_{M Q}\right)\left(r_{\mathrm{NQ}}\right) p_{1}$ \\
& & $Q_{1} / Q_{2}$ & $\frac{r_{M Q}\left(1-r_{N Q}\right)}{r_{M N}}$ & $p_{3}$ & $\frac{r_{M Q}\left(1-r_{N Q}\right)}{r_{M N}} p_{3}$ & $\frac{1}{2} r_{M Q}\left(1-r_{N Q}\right) p_{3}$ \\
$M_{1} N_{1} / M_{2} N_{1}$ & \multirow{2}{*}{$r_{M N}$} & $Q_{1} / Q_{1}$ & $\frac{\left(r_{M Q}\right)\left(1-r_{N Q}\right)}{r_{M N}}$ & $p_{1}$ & $\frac{\left(r_{M Q}\right)\left(1-r_{N Q}\right)}{r_{M N}} p_{1}$ & $\frac{1}{2}\left(r_{M Q}\right)\left(1-r_{N Q}\right) p_{1}$ \\
& & $Q_{1} / Q_{2}$ & $\frac{\left(r_{N Q)}\right)\left(1-r_{M Q}\right)}{r_{M N}}$ & $p_{3}$ & $\frac{\left(r_{N Q}\right)\left(1-r_{M Q}\right)}{r_{M N}} p_{3}$ & $\frac{1}{2}\left(r_{N Q}\right)\left(1-r_{M Q}\right) p_{3}$ \\
$M_{1} N_{1} / M_{2} N_{2}$ & \multirow{2}{*}{$\left(1-r_{M N}\right)$} & $Q_{1} / Q_{1}$ & $\frac{\left(r_{M Q}\right)\left(r_{M Q}\right)}{1-r_{M N}}$ & $p_{1}$ & $\frac{\left(r_{M Q}\right)\left(r_{N Q}\right)}{1-r_{M N}} p_{1}$ & $\frac{1}{2}\left(r_{M Q}\right)\left(r_{N Q}\right) p_{1}$ \\
& & $Q_{1} / Q_{2}$ & $\frac{\left(1-r_{M Q}\right)\left(1-r_{N Q}\right)}{1-r_{M N}}$ & $p_{3}$ & $\frac{\left(1-r_{M Q}\right)\left(1-r_{N Q}\right)}{1-r_{M N}} p_{3}$ & $\frac{1}{2}\left(1-r_{M Q}\right)\left(1-r_{N Q}\right) p_{3}$ \\
\hline \hline
\end{tabular}

standard tests of the regression parameters $\beta_{1}=0$ and $\beta_{2}=0$ in terms of $r_{M Q}, p_{1}, p_{2}$ and $p_{3}$, where

$$
\begin{aligned}
\mu_{M_{1} / M_{2}}-\mu_{M_{1} / M_{1}}= & \left(1-2 r_{M Q}\right)\left[r_{M Q}\left(p_{1}-2 p_{3}+p_{2}\right)\right. \\
& \left.+\left(p_{3}-p_{1}\right)\right] \\
\mu_{M_{2} / M_{2}}-\mu_{M_{1} / M_{1}}= & \left(1-2 r_{M Q}\right)\left(p_{2}-p_{1}\right) .
\end{aligned}
$$

In this model, the test of the regression coefficient $\beta_{1}=0$ is a test of $r_{M Q}=0.50$ and $p_{1}=p_{2}=p_{3}$, and $\beta_{2}=0$ is a test of $r_{M Q}=0.50$ and $p_{1}=p_{2}$. Assuming that $p_{1} \neq p_{2}$, both tests are a direct test of $r_{M Q}=0.50$. The test of $\beta_{2}$ in the $\mathrm{F}_{2}$ is conceptually equivalent to the test of $\beta_{1}$ in the backcross.

\section{(b) Logistic model}

The logistic model has been suggested in binary trait analysis. The logistic regression model is written as:

$Y_{i}=\pi\left(X_{i}\right)+\epsilon_{i} ; \quad i=1, \ldots, n$

where for a backcross, single BTL, single marker model

$\pi\left(X_{i}\right)=\frac{\exp \left(\beta_{0}+\beta_{1} X_{i}\right)}{1+\exp \left(\beta_{0}+\beta_{1} X_{i}\right)}$,

and $X_{i}$ is the marker class as defined in (1).

For each trait and marker status classification we derived $\pi\left(X_{i}=0\right)$ and $\pi\left(X_{i}=1\right)$, giving rise to the $\log$ odds ratio

$\beta_{1}=\ln \left[\frac{\pi\left(X_{i}=1\right)}{1-\pi\left(X_{i}=1\right)}\right] / \ln \left[\frac{\pi\left(X_{i}=0\right)}{1-\pi\left(X_{i}=0\right)}\right]$

(Hosmer \& Lemeshow, 1989; Agresti, 1990). This expression lends interpretability to the model, $\beta_{1}$ now represents a comparative assessment of the odds among individuals with the trait $(Y=1)$ and marker genotype $X=1$, compared with the odds among individuals with the trait and marker genotype $X=0$.

Applying the probability model derived above (Table 1), the expected values of the log odds are

$e^{\beta_{0}}=\left[\frac{p_{1}-r_{M Q} p_{1}+r_{M Q} p_{3}}{1-\left(p_{1}-r_{M Q} p_{1}+r_{M Q} p_{3}\right)}\right]$

and

$e^{\beta_{1}}=\left[\frac{r_{M Q} p_{1}+p_{3}-r_{M Q} p_{3}}{1-\left(r_{M Q} p_{1}+p_{3}-r_{M Q} p_{3}\right)}\right] / e^{\beta_{0}}$.

The logistic regression model for an $\mathrm{F}_{2}$, single BTL, single marker model is written as:

$\pi(\mathbf{X})=\frac{\exp \left(\beta_{0}+\beta_{1} X_{1 i}+\beta_{2} X_{2 i}\right)}{1+\exp \left(\beta_{0}+\beta_{1} X_{1 i}+\beta_{2} X_{2 i}\right)}$

where $X_{1 i}$ and $X_{2 i}$ are indicator variables as defined in (1). 
As in the backcross, the expected log odds for $M_{1} M_{1}\left(e^{\beta_{0}}\right), M_{1} M_{2}\left(e^{\beta_{1}}\right)$ and $M_{2} M_{2}\left(e^{\beta_{2}}\right)$ are derived from the conditional probabilities in Table 2 (see Appendix).

The tests of the regression parameters in the logistic model are tests of $\beta_{i}=1$ as they are a ratio of means, compared with the linear regression which is a difference between means. The tests in the logistic regression have the same interpretation as the tests in the linear regression.

\section{(iv) Power}

The power of linear and logistic regression coefficient tests to detect BTL were directly compared. The tests between the regression models were evaluated in terms of their power to detect BTL under a variety of genetic models in the backcross and the $F_{2}$ experimental designs. We computed $P$ values via permutation (Churchill \& Doerge, 1994; Doerge \& Churchill, 1996) to ensure that the type I error was valid and was comparable between the two regression strategies. As mentioned previously, with our parameterization, the null hypothesis tests of the $\beta_{i}$ are the same in the linear and logistic models. Therefore, we considered the regression model with the higher power of the tests to be the better method of implementation.

\section{(v) Estimation of recombination and penetrance}

Once a BTL is detected, the distance from the marker to the BTL can be estimated. Currently, recombination can be estimated directly from the observed trait and marker data. The unadjusted estimate is $\hat{r}_{M Q}=n_{r} / n$, where $n_{r}$ is the number of individuals with the trait and marker type that are discordant according to the genetic model, and $n$ is the total number of individuals (Lynch \& Walsh, 1998). This estimate does not allow for reduced penetrance and implicitly assumes that $p_{1}=0$ and $p_{2}=1$.

We derive moment estimators for recombination that are adjusted for penetrance in both the backcross and $\mathrm{F}_{2}$ experimental designs assuming $p_{1}$ and/or $p_{2}$ are known. In the backcross, the moment estimators are constructed using the equations for the marker means in the backcross, where

$\mu_{M_{1} / M_{1}}=\left(1-r_{M Q}\right) p_{1}+r_{M Q} p_{3}$

$\mu_{M_{1} / M_{2}}=r_{M Q} p_{1}+\left(1-r_{M Q}\right) p_{3}$.

The first equation was solved for $r_{M Q}$ giving the estimate

$r_{M Q}=\frac{p_{1}-\mu_{M_{1} / M_{1}}}{p_{1}-p_{3}}$

We then substituted this estimate of $r_{M Q}$ into the second equation and solved for $p_{3}$ giving the estimate $p_{3}=\mu_{M_{1} / M_{2}}+\mu_{M_{1} / M_{1}}-p_{1}$. We then substituted this estimate back into the equation for $r_{M Q}$, resulting in the estimate

$r_{M Q}=\frac{p_{1}-\mu_{M_{1} / M_{1}}}{2 p_{1}-\mu_{M_{1} / M_{2}}-\mu_{M_{1} / M_{1}}}$.

Similarly, moments estimates for the $\mathrm{F}_{2}$ experimental design were constructed (Table 4 ).

When $p_{1}=0$ and $p_{3}=1$ in the backcross, the adjusted estimate of $r_{M Q}$ reduces to the unadjusted estimate. Estimates of $p_{3}$ are also important as they give insight into the genetic model (Table 4). The moment estimator for $p_{3}$ was derived assuming that $p_{1}$ and $p_{2}$ were known. The estimates of $r_{M Q}$ and $p_{3}$ depend upon $p_{1}$ and $p_{2}$, and if $p_{1}$ and $p_{2}$ are known exactly, then the moment estimators of $r_{M Q}$ and $p_{3}$ are unbiased. However, in experimental situations the precise values of $p_{1}$ and $p_{2}$ may not be known and, therefore, we evaluated the sensitivity of the estimates of $r_{M Q}$ and $p_{3}$ to misspecification of $p_{1}$ and $p_{2}$.

\section{(vi) Two markers}

We now extend our parameterization to include a second marker. For a two marker backcross design, with a map $M Q N$, the joint and conditional probability distributions can be described in terms $r_{M Q}$, $r_{N Q}, r_{M N}, p_{1}$ and $p_{3}$. There are four observable marker genotypes, and two unobservable BTL genotypes within each marker type. The full joint and conditional probability distributions are given in Table 3 .

In order to derive moments estimators we assumed $r_{M N}$ and $p_{1}$ were known. We then used the method of moments, as described for the single marker case, to derive moment estimators for $r_{M Q}, r_{N Q}$ and $p_{3}$. These estimators are as follows:

$$
\begin{aligned}
\hat{p}_{3}= & \left(\mu_{M_{2} N_{1}}+\mu_{M_{1} N_{2}}-\mu_{M_{2} N_{2}}-\mu_{M_{1} N_{1}}\right) \\
& \left.\times r_{M N}+\mu_{M_{2} N_{2}}+\mu_{M_{1} N_{1}}-p_{1}\right) \\
\hat{r}_{M Q}= & \frac{p_{1}-\left(\mu_{M_{1} N_{2}}-\mu_{M_{1} N_{1}}\right) r_{M N}-\mu_{M_{1} N_{1}}}{p_{3}-p_{1}} \\
\hat{r}_{N Q}= & \frac{p_{1}-\left(\mu_{M_{2} N_{1}}-\mu_{M_{1} N_{1}}\right) r_{M N}-\mu_{M_{1} N_{1}}}{p_{3}-p_{1}} .
\end{aligned}
$$

\section{(vii) Comparison with interval regression}

Interval regression has been described in detail by Martinez \& Curow (1992) for the backcross and by Haley \& Knott (1992) for the $F_{2}$. Briefly, this approach uses flanking markers to define the coefficients of the regression as mean, additive or dominance effects. For $s$ steps along the interval between markers $M$ and $N$ values of $X$ are calculated according to the conditional probability of a QTL in that location. In the backcross design, at each position in the interval, the estimated regression parameter $\hat{\beta}_{1}$ provides an estimate of the 


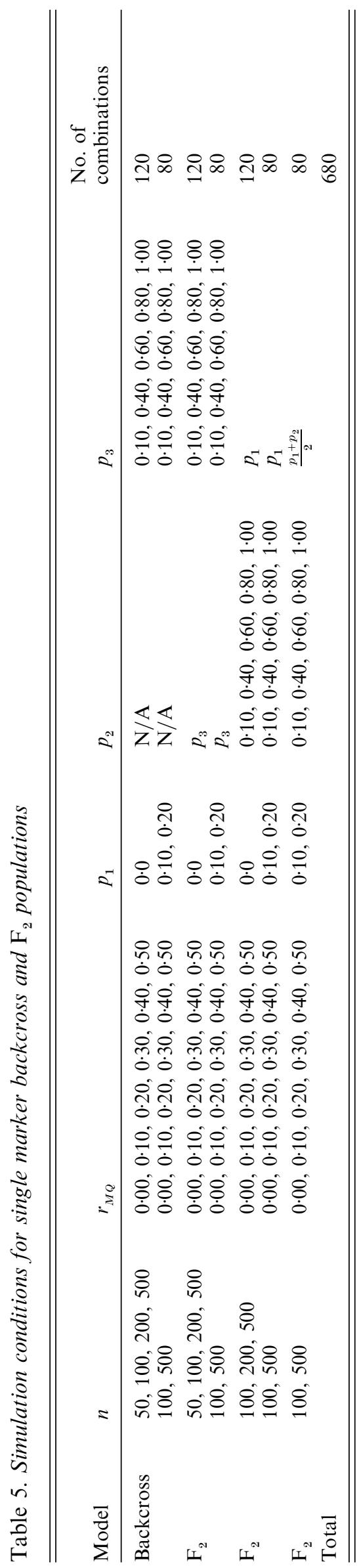

additive genetic effect $a$ for a specific $r_{M Q}$. Thus, the test of the regression parameter $\beta_{1}=0$ in interval regression is the test $a=0$ for a specific $r_{M Q}$. This is a statistical test for an additive effect. Similarly, in the $\mathrm{F}_{2}$, the tests of the regression coefficients in interval regression are tests of additive and dominance effect. The test $\beta_{1}=0$ is the test $a=0$ and the test $\beta_{2}=0$ is the test $d=0$. The test of the entire model is a test of $a+d=0$. Using our notation, the test $a+d=0$ corresponds to a test of $p_{3}-p_{1}=0$.

The interval parameterization thus provides a mechanism to test for effect using tests of the regression parameters. In our parameterization, the regression coefficients are tests for detection. Thus, the two parameterizations have different null hypotheses for the tests of the regression coefficients and are not comparable in terms of power. However, the estimates of $r_{M Q}$ and $p_{3}$ produced by both parameterizations are comparable.

\section{(viii) Simulations}

Data were simulated for the single marker backcross, two marker backcross, and single marker $F_{2}$ experimental frameworks for the cases given in Table 5. There were a total of 680 combinations of parameters simulated. For each combination of parameters, 1000 replicates of the simulation were performed. For each replicate, the null hypothesis was rejected when the empirical $P$ value for that replicate was less than the nominal alpha, $0 \cdot 05$. For each simulation (set of 1000 replicates), the power of the linear model was compared with that of the logistic model using McNemar's test (Agresti, 1990). The power for each test of a regression parameter was estimated as the number of times the empirical $P$ value for that replicate was less than $0 \cdot 05$ divided by the number of replicates.

For each replicate, recombination was estimated using the unadjusted $\hat{r}_{M Q}=n_{r} / n$ and adjusted estimates, and $p_{3}$ was estimated (Table 4 ). The adjusted estimates assumed $p_{1}$ and $p_{2}$ were known and the sensitivity of the adjusted $r_{M Q}$ and $p_{3}$ estimates to misspecification of $p_{1}$ and $p_{2}$ was examined by setting $p_{1}$ and $p_{2}$ to incorrect values in the estimation of $r_{M Q}$ and $p_{3}$. Incorrect values ranged across all possible values of $p_{1}$ and $p_{2}$ consistent with the initial assumption $p_{1}<p_{2}$.

Two marker backcross populations were simulated for the cases given in Table 6. For each replicate, $r_{M Q}$ and $p_{3}$ were estimated using the moment estimators derived from our single marker model, from our two marker model, and using the interval regression method. For the interval regression method, we stepped through the interval from $r_{M Q}=0.00$ to $r_{M Q}=\hat{r}_{M N}$ using increments of 0.005. We selected the value of $r_{M Q}$ in the interval from 0.00 to $r_{M N}$ 
that produced the lowest value for the approximate likelihood ratio test statistic

$$
\left(n * \log \frac{S S E_{R}}{S S E_{F}}\right)
$$

where $S S E_{R}$ is the sum of squared errors for the reduced model, and $S S E_{F}$ is the sum of squared errors for the full model.

\section{Results}

\section{(i) Power}

In the backcross, power for the test of $\beta_{1}$ was similar in the linear and logistic regression models except when the marker was close to the BTL $\left(r_{M Q}<0 \cdot 10\right)$, the difference between the parental lines was small $\left(\left(p_{3}-p_{1}\right)<0 \cdot 4\right)$ and the sample size was small. In these cases, the linear model had significantly better power than the logistic model ( $p \leqslant 0 \cdot 05$, McNemar's test). Similarly, in the $F_{2}$ the power for the logistic model test of the $\beta_{2}$ was significantly lower than that of the linear model $(P \leqslant 0 \cdot 05$, McNemar's test $)$ under similar conditions. Additionally, examining the power curves for $\beta_{2}$ under different values of recombination for these conditions revealed that power was not monotonic for the logistic regression (Fig. 2).

Power also depends on the absolute difference in the penetrance of the parental lines $\left(p_{2}-p_{1}\right)$. As mentioned in Section 2, we assumed that the penetrance of the parental lines was different, which made the tests of the $\beta_{i}$ simple tests of the recombination between marker and trait. We found that the power of the tests of the $\beta_{i}=0$ depended upon maximizing the difference in the parental lines (Fig. 3). The larger the difference between the parental lines, the higher the power.

We can derive the relationship between the power of the test and the difference between parental lines by examining the expected values of $\beta_{2}$ in the linear model. In the $\mathrm{F}_{2}$ design, the $E\left(\beta_{2}\right)=$ $\left(1-2 r_{M Q}\right)\left(p_{2}-p_{1}\right)$. When the parental lines are equal $\left(p_{2}-p_{1}=0\right)$, the $E\left(\beta_{2}\right)=0$ and the power of the test is the nominal alpha level specified. As the difference between the parents increases, the expected value moves further from zero for a fixed value of $r_{M Q}$. Correspondingly, the permuted power for the test of $\beta_{2}$ from our simulations increased as the difference in the parental lines increased (Fig. 3). Similarly, power for the test of $\beta_{1}$ in the backcross decreased monotonically as the difference $\left(p_{3}-p_{1}\right)$ decreased. Our simulations showed that as the difference in the means between parental and $F_{1}$ lines increased, power increased.

Power for tests on $\beta_{1}$ in the backcross and $\beta_{2}$ in the $\mathrm{F}_{2}$ in the linear regression framework increased as linkage between marker and BTL increased, or as 


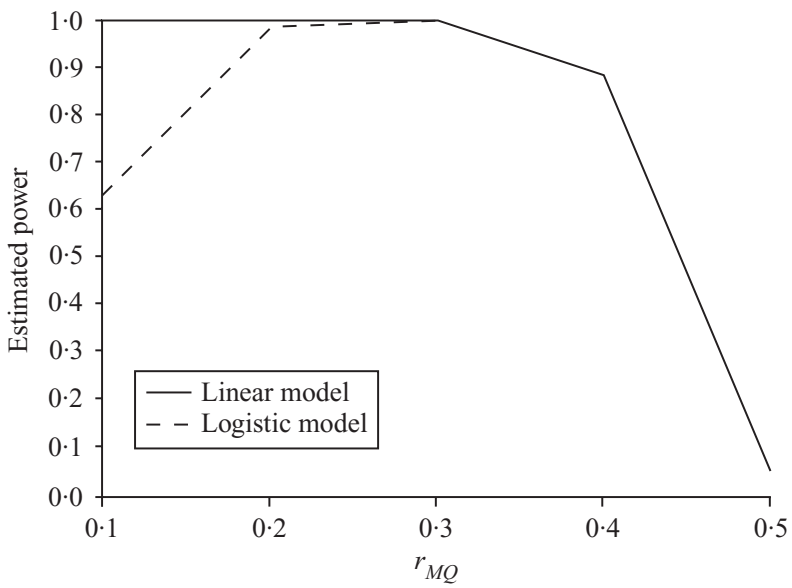

Fig. 2. Power of the test of $\beta_{1}$ in linear and logistic models as a function of recombination.

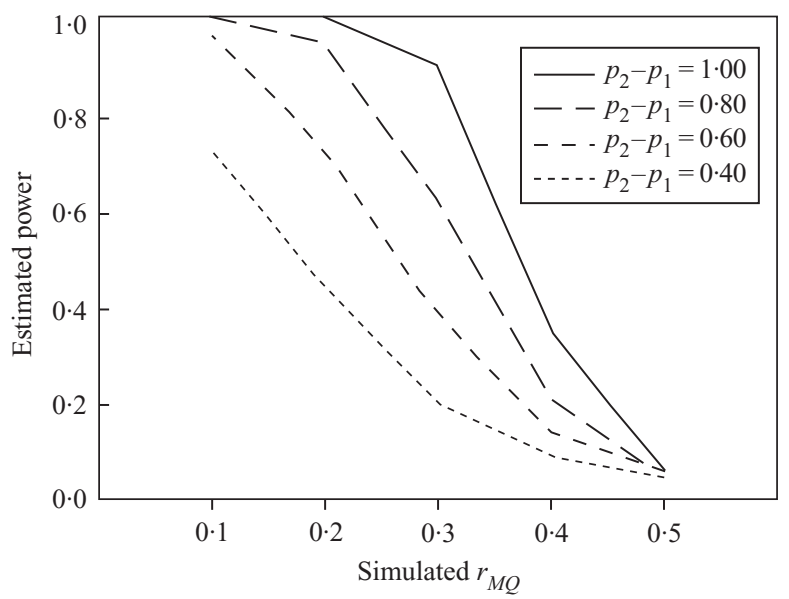

Fig. 3. Power in the linear model as a function of recombination and difference between parental lines.

recombination between marker and BTL decreased (Fig. 3). Thus, the closer a marker to the true BTL location the higher the power for detecting the BTL. Power also increased as sample size increased.

The power of the test of $\beta_{1}$ in the $\mathrm{F}_{2}$ depends on the relationship between the $F_{1}$ and the parental lines which is described by the genetic model. However, if we define our indicator variables differently than what is shown in (1), the test of $\beta_{1}$ and the power of the test of $\beta_{1}$ will be different. For example, if $X_{2 i}=1$ when $M T=M_{1} / M_{1}$ instead of when $M T=M_{2} / M_{2}$, the $\quad E\left(\beta_{1}\right)=\mu_{M_{1} / M_{2}}-\mu_{M_{2} / M_{2}}=\left(1-2 r_{M Q}\right)\left[r_{M Q}\left(p_{1}-\right.\right.$ $\left.\left.2 p_{3}+p_{2}\right)+\left(p_{3}-p_{2}\right)\right]$. This demonstrates the importance of careful specification of the indicator variables and the impact of the specification on power for tests of $\beta_{1}$ in the $\mathrm{F}_{2}$.

\section{(ii) Estimates of $r_{M Q}$ and $p_{3}$}

In the backcross, estimates of $r_{M Q}$ unadjusted for penetrance $\left(n_{r} / n\right)$ are unbiased only when Parent ${ }_{1}$ had no individuals expressing the trait $\left(p_{1}=0\right)$ and the $\mathrm{F}_{1}$

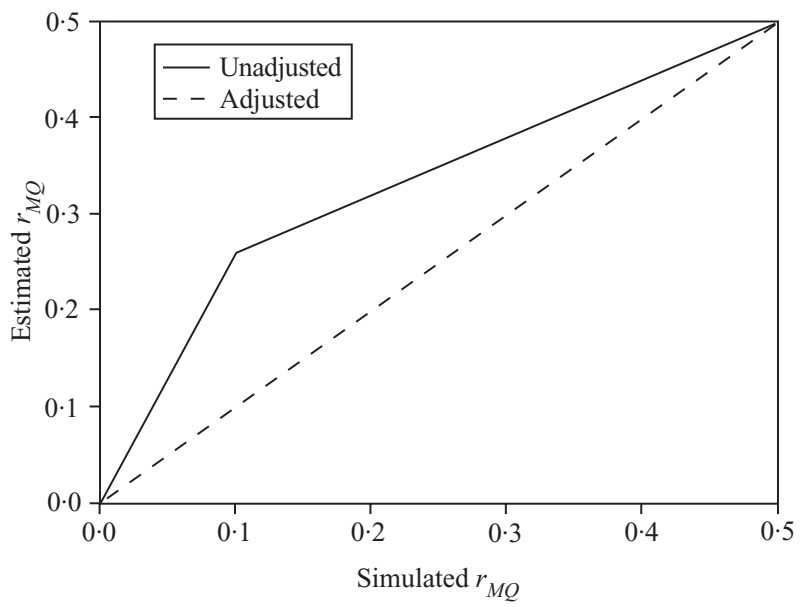

Fig. 4. Estimation of recombination unadjusted for incomplete penetrance compared with estimation of recombination adjusted for incomplete penetrance.

had all individuals expressing the trait $\left(p_{3}=1\right)$. As penetrance parameters moved away from these extreme values, the estimate of recombination using this formulation became increasingly biased (Fig. 4). In contrast, estimates of $r_{M Q}$ adjusted for penetrance (Table 4) were unbiased.

In the $\mathrm{F}_{2}$, the adjusted $r_{M Q}$ estimates (Table 4) and $p_{3}$ estimates were unbiased when $p_{1}$ and $p_{2}$ are specified correctly as shown in a subset of the simulation runs in Tables 7-10. As sample size decreased, the standard errors of the estimates for $r_{M Q}$ and $p_{3}$ increased, and as the difference between parental lines decreased $\left(p_{2}-p_{1}\right)$, the standard errors for the estimates of $r_{M Q}$ increased.

The estimates of both $r_{M Q}$ and $p_{3}$ were biased when the values of $p_{1}$ and $p_{2}$ were misspecified. For estimates of $r_{M Q}$, if the penetrance of Parent ${ }_{1}\left(p_{1}\right)$ was specified too low (further from $p_{2}$ ), then estimates were biased towards 0.50 while if $p_{1}$ is specified too high (closer to $p_{2}$ ), estimates were biased towards zero (Fig. $5 a$ ). If misspecification was large, then estimates of $r_{M Q}$ were sometimes greater than 0.50 or less than 0.00 . However, when $r_{M Q}=0.50$ the estimated value was approximately 0.50 regardless of the misspecification of $p_{1}$. Results were similar for misspecification of $p_{2}$ (Fig. $5 c$ ). In both cases, misspecification of less than approximately $20 \%$ resulted in estimates of $r_{M Q}$ reasonably close to the true value even for samples of size 100 . For estimates of $p_{3}$, if $p_{1}$ was specified too low (further from $p_{2}$ ), then estimates were biased towards $p_{2}$, while if $p_{1}$ was specified too high (closer to $p_{2}$ ), estimates were biased towards $p_{1}$ (Fig. $5 b$ ). Results were similar for misspecification of $p_{2}$ (Fig. $5 d$ ).

\section{(iii) Two markers}

The estimates and the standard error of the estimates of $r_{M Q}$ were not improved by expanding our para- 
Table 7. Estimates of recombination parameter $\left(\hat{r}_{M Q}\right)$ and penetrance parameter $\left(\hat{p}_{3}\right)$ for data simulated for an $\mathrm{F}_{2}$, dominant model, $\left(p_{1}=0\right.$, and $\left.p_{2}=p_{3}\right), n=100$

\begin{tabular}{|c|c|c|c|c|c|}
\hline$p_{1}$ & $p_{2}$ & $p_{3}$ & $r_{M Q}$ & $\hat{p}_{3}\left(\operatorname{se}\left(\hat{p}_{3}\right)\right)$ & $\hat{r}_{M Q}\left(\operatorname{se}\left(\hat{r}_{M Q}\right)\right)$ \\
\hline \multirow[t]{5}{*}{$0 \cdot 00$} & $1 \cdot 00$ & $1 \cdot 00$ & $0 \cdot 10$ & $0.9997(0 \cdot 001866)$ & $0 \cdot 1004(0 \cdot 001341)$ \\
\hline & & & $0 \cdot 20$ & $0.9975(0.002297)$ & $0 \cdot 1996(0 \cdot 001654)$ \\
\hline & & & $0 \cdot 30$ & $0.997(0.002549)$ & $0 \cdot 2996(0 \cdot 001938)$ \\
\hline & & & $0 \cdot 40$ & $1.003(0.002736)$ & $0 \cdot 4022(0 \cdot 002014)$ \\
\hline & & & $0 \cdot 50$ & $0.9947(0 \cdot 002723)$ & $0.501(0.002004)$ \\
\hline \multirow[t]{5}{*}{$0 \cdot 00$} & $0 \cdot 80$ & $0 \cdot 80$ & $0 \cdot 10$ & $0 \cdot 8004(0 \cdot 002671)$ & $0 \cdot 1007(0 \cdot 002112)$ \\
\hline & & & $0 \cdot 20$ & $0 \cdot 8013(0 \cdot 002893)$ & $0 \cdot 2048(0 \cdot 002427)$ \\
\hline & & & $0 \cdot 30$ & $0 \cdot 8012(0 \cdot 003007)$ & $0.3045(0.002732)$ \\
\hline & & & $0 \cdot 40$ & $0 \cdot 8067(0.003154)$ & $0 \cdot 4015(0 \cdot 002793)$ \\
\hline & & & $0 \cdot 50$ & $0 \cdot 7958(0 \cdot 003125)$ & $0.5036(0.002743)$ \\
\hline \multirow[t]{5}{*}{$0 \cdot 00$} & $0 \cdot 60$ & $0 \cdot 60$ & $0 \cdot 10$ & $0 \cdot 6014(0 \cdot 002915)$ & $0.0927(0.003113)$ \\
\hline & & & $0 \cdot 20$ & $0.5945(0.003076)$ & $0 \cdot 2084(0 \cdot 003378)$ \\
\hline & & & $0 \cdot 30$ & $0.5935(0 \cdot 003112)$ & $0 \cdot 3008(0 \cdot 00358)$ \\
\hline & & & $0 \cdot 40$ & $0 \cdot 6008(0 \cdot 003163)$ & $0 \cdot 3949(0.003675)$ \\
\hline & & & $0 \cdot 50$ & $0 \cdot 6004(0 \cdot 00318)$ & $0 \cdot 4954(0 \cdot 003864)$ \\
\hline \multirow[t]{5}{*}{$0 \cdot 00$} & $0 \cdot 40$ & $0 \cdot 40$ & $0 \cdot 10$ & $0 \cdot 4004(0 \cdot 002844)$ & $0 \cdot 1043(0 \cdot 004436)$ \\
\hline & & & $0 \cdot 20$ & $0 \cdot 4041(0 \cdot 002839)$ & $0 \cdot 2063(0 \cdot 00488)$ \\
\hline & & & $0 \cdot 30$ & $0.3965(0 \cdot 002938)$ & $0 \cdot 3057(0 \cdot 004895)$ \\
\hline & & & $0 \cdot 40$ & $0 \cdot 394(0 \cdot 002834)$ & $0 \cdot 4074(0 \cdot 005231)$ \\
\hline & & & $0 \cdot 50$ & $0 \cdot 3961(0 \cdot 002762)$ & $0 \cdot 4935(0.005119)$ \\
\hline
\end{tabular}

Table 8. Estimates of recombination parameter $\left(\hat{r}_{M Q}\right)$ and penetrance parameter $\left(\hat{p}_{3}\right)$ for data simulated for an $\mathrm{F}_{2}$, recessive model, $\left(p_{1}=p_{3}=0\right), n=100$

\begin{tabular}{|c|c|c|c|c|c|}
\hline$p_{1}$ & $p_{2}$ & $p_{3}$ & $r_{M Q}$ & $\hat{p}_{3}\left(\operatorname{se}\left(\hat{p}_{3}\right)\right)$ & $\hat{r}_{M Q}\left(\operatorname{se}\left(\hat{r}_{M Q}\right)\right)$ \\
\hline \multirow[t]{5}{*}{$0 \cdot 00$} & $1 \cdot 00$ & $0 \cdot 00$ & $0 \cdot 10$ & $0 \cdot 002871(0 \cdot 001821)$ & $0.09887(0.001331)$ \\
\hline & & & $0 \cdot 20$ & $-0.003263(0.002406)$ & $0 \cdot 1994(0 \cdot 001618)$ \\
\hline & & & $0 \cdot 30$ & $-0.001751(0.002611)$ & $0 \cdot 3028(0 \cdot 001842)$ \\
\hline & & & $0 \cdot 40$ & $2 \cdot 354 \mathrm{e}-05(0 \cdot 002739)$ & $0 \cdot 4028(0 \cdot 001927)$ \\
\hline & & & $0 \cdot 50$ & $-0.00419(0.002773)$ & $0 \cdot 5002(0 \cdot 001977)$ \\
\hline \multirow[t]{5}{*}{$0 \cdot 00$} & $0 \cdot 80$ & $0 \cdot 00$ & $0 \cdot 10$ & $0 \cdot 001787(0 \cdot 001998)$ & $0.09931(0.00198)$ \\
\hline & & & $0 \cdot 20$ & $0.002596(0.002282)$ & $0 \cdot 1984(0 \cdot 00213)$ \\
\hline & & & $0 \cdot 30$ & $0.003764(0.002443)$ & $0 \cdot 2992(0 \cdot 002276)$ \\
\hline & & & $0 \cdot 40$ & $0.002801(0 \cdot 00241)$ & $0 \cdot 4021(0 \cdot 002206)$ \\
\hline & & & $0 \cdot 50$ & $0.001556(0 \cdot 002608)$ & $0 \cdot 5006(0 \cdot 002239)$ \\
\hline \multirow[t]{5}{*}{$0 \cdot 00$} & $0 \cdot 60$ & $0 \cdot 00$ & $0 \cdot 10$ & $-0.0007081(0 \cdot 001866)$ & $0 \cdot 1016(0 \cdot 002641)$ \\
\hline & & & $0 \cdot 20$ & $-0.000394(0.002061)$ & $0 \cdot 2029(0 \cdot 002688)$ \\
\hline & & & $0 \cdot 30$ & $-0.001148(0.002142)$ & $0 \cdot 3007(0 \cdot 002785)$ \\
\hline & & & $0 \cdot 40$ & $-0.0007598(0.002215)$ & $0 \cdot 3964(0.00272)$ \\
\hline & & & $0 \cdot 50$ & $-9 \cdot 109 \mathrm{e}-05(0.002246)$ & $0 \cdot 4992(0 \cdot 002781)$ \\
\hline \multirow[t]{5}{*}{$0 \cdot 00$} & $0 \cdot 40$ & $0 \cdot 00$ & $0 \cdot 10$ & $-0.0003342(0.001709)$ & $0.09977(0.003703)$ \\
\hline & & & $0 \cdot 20$ & $0.0002425(0.001831)$ & $0 \cdot 1963(0 \cdot 003605)$ \\
\hline & & & $0 \cdot 30$ & $-0.0009838(0.001816)$ & $0 \cdot 3004(0.00343)$ \\
\hline & & & $0 \cdot 40$ & $-0.001232(0 \cdot 001905)$ & $0.404(0.003402)$ \\
\hline & & & $0 \cdot 50$ & $0.0001936(0.001873)$ & $0 \cdot 4976(0 \cdot 003295)$ \\
\hline
\end{tabular}

meterization to include the second marker (Table 11). However, it is likely that additional markers will help position the BTL to the left or right of the primary marker due to the gain in available information provided by these markers. (iv) Comparison with interval regression

The estimates of $r_{M Q}$ and the standard errors of $r_{M Q}$ were similar between the interval regression and our estimators for both the single and two marker 
Table 9. Estimates of recombination parameter $\left(\hat{r}_{M Q}\right)$ and penetrance parameter $\left(\hat{p}_{3}\right)$ for data simulated for an $\mathrm{F}_{2}, p_{3}=\frac{p_{1}+p_{2}}{2}, n=100$

\begin{tabular}{|c|c|c|c|c|c|}
\hline$p_{1}$ & $p_{2}$ & $p_{3}$ & $r_{M Q}$ & $\hat{p}_{3}\left(\operatorname{se}\left(\hat{p}_{3}\right)\right)$ & $\hat{r}_{M Q}\left(\operatorname{se}\left(\hat{r}_{M Q}\right)\right)$ \\
\hline \multirow[t]{5}{*}{$0 \cdot 10$} & $1 \cdot 00$ & $0 \cdot 55$ & $0 \cdot 10$ & $0 \cdot 5519(0 \cdot 002689)$ & $0 \cdot 1007(0 \cdot 001667)$ \\
\hline & & & $0 \cdot 20$ & $0 \cdot 5507(0 \cdot 002972)$ & $0 \cdot 2014(0 \cdot 002064)$ \\
\hline & & & $0 \cdot 30$ & $0.5449(0.00315)$ & $0 \cdot 3021(0 \cdot 002391)$ \\
\hline & & & $0 \cdot 40$ & $0.549(0.003237)$ & $0.3992(0.002541)$ \\
\hline & & & $0 \cdot 50$ & $0.5492(0.003271)$ & $0.5016(0.002478)$ \\
\hline \multirow[t]{5}{*}{$0 \cdot 10$} & $0 \cdot 80$ & $0 \cdot 45$ & $0 \cdot 10$ & $0.4473(0 \cdot 002963)$ & $0 \cdot 1017(0 \cdot 002598)$ \\
\hline & & & $0 \cdot 20$ & $0.4537(0.003002)$ & $0 \cdot 2063(0 \cdot 002893)$ \\
\hline & & & $0 \cdot 30$ & $0 \cdot 4538(0 \cdot 003117)$ & $0 \cdot 2907(0 \cdot 003097)$ \\
\hline & & & $0 \cdot 40$ & $0 \cdot 4521(0 \cdot 003216)$ & $0.3979(0.003169)$ \\
\hline & & & $0 \cdot 50$ & $0 \cdot 4444(0 \cdot 003172)$ & $0.497(0.003235)$ \\
\hline \multirow[t]{5}{*}{$0 \cdot 10$} & 0.60 & $0 \cdot 35$ & $0 \cdot 10$ & $0 \cdot 3496(0 \cdot 002865)$ & $0 \cdot 1006(0 \cdot 003822)$ \\
\hline & & & $0 \cdot 20$ & $0 \cdot 3482(0 \cdot 00291)$ & $0 \cdot 201(0 \cdot 004081)$ \\
\hline & & & $0 \cdot 30$ & $0 \cdot 3459(0 \cdot 002985)$ & $0 \cdot 2994(0 \cdot 004262)$ \\
\hline & & & $0 \cdot 40$ & $0 \cdot 3531(0 \cdot 003113)$ & $0.3972(0.004255)$ \\
\hline & & & $0 \cdot 50$ & $0 \cdot 35(0 \cdot 003052)$ & $0.4958(0.004339)$ \\
\hline
\end{tabular}

Table 10. Estimates of recombination parameter $\left(\hat{r}_{M Q}\right)$ and penetrance parameter $\left(\hat{p}_{3}\right)$ for data simulated for an $\mathrm{F}_{2}, p_{1}=p_{3}, n=100$

\begin{tabular}{|c|c|c|c|c|c|}
\hline$p_{1}$ & $p_{2}$ & $p_{3}$ & $r_{M Q}$ & $\hat{p}_{3}\left(\operatorname{se}\left(\hat{p}_{3}\right)\right)$ & $\hat{r}_{M Q}\left(\operatorname{se}\left(\hat{r}_{M Q}\right)\right)$ \\
\hline \multirow[t]{5}{*}{$0 \cdot 10$} & $1 \cdot 00$ & $0 \cdot 10$ & $0 \cdot 10$ & $0 \cdot 1009(0 \cdot 002401)$ & $0 \cdot 1022(0 \cdot 001785)$ \\
\hline & & & $0 \cdot 20$ & $0 \cdot 1005(0 \cdot 00265)$ & $0 \cdot 2008(0 \cdot 002067)$ \\
\hline & & & $0 \cdot 30$ & $0 \cdot 101(0 \cdot 002862)$ & $0 \cdot 2999(0 \cdot 002258)$ \\
\hline & & & $0 \cdot 40$ & $0 \cdot 09833(0 \cdot 002953)$ & $0 \cdot 4012(0 \cdot 002267)$ \\
\hline & & & $0 \cdot 50$ & $0.09954(0.00297)$ & $0.4983(0.002344)$ \\
\hline \multirow[t]{5}{*}{$0 \cdot 10$} & $0 \cdot 80$ & $0 \cdot 10$ & $0 \cdot 10$ & $0 \cdot 1006(0 \cdot 00244)$ & $0.09807(0 \cdot 00257)$ \\
\hline & & & $0 \cdot 20$ & $0 \cdot 1011(0 \cdot 002732)$ & $0 \cdot 2005(0 \cdot 002673)$ \\
\hline & & & $0 \cdot 30$ & $0.0992(0.00275)$ & $0 \cdot 2998(0.00282)$ \\
\hline & & & $0 \cdot 40$ & $0.09831(0 \cdot 002709)$ & $0 \cdot 395(0 \cdot 002878)$ \\
\hline & & & $0 \cdot 50$ & $0 \cdot 1007(0 \cdot 002945)$ & $0 \cdot 499(0 \cdot 003015)$ \\
\hline \multirow[t]{5}{*}{$0 \cdot 10$} & 0.60 & $0 \cdot 10$ & $0 \cdot 10$ & $0 \cdot 105(0 \cdot 00241)$ & $0.09658(0.003651)$ \\
\hline & & & $0 \cdot 20$ & $0.09976(0 \cdot 002629)$ & $0 \cdot 1952(0 \cdot 003741)$ \\
\hline & & & $0 \cdot 30$ & $0 \cdot 1021(0 \cdot 002674)$ & $0.2951(0.003782)$ \\
\hline & & & $0 \cdot 40$ & $0.09402(0 \cdot 002611)$ & $0.4038(0.003753)$ \\
\hline & & & $0 \cdot 50$ & $0.09876(0 \cdot 002642)$ & $0 \cdot 4991(0 \cdot 00376)$ \\
\hline
\end{tabular}

simulations (Table 11). Estimates of $p_{3}$ were also comparable across all three approaches. When the flanking markers were unlinked $r_{M N}=0.50$, the interval regression tended to produced biased results.

\section{Discussion and conclusions}

We have described a simple parameterization that can detect and localize BTL in plant and animal populations for backcross and $\mathrm{F}_{2}$ experiments. The method relies on simple linear regression combined with a permutation algorithm that can be easily applied and implemented in commercially available software such as SAS or Splus with little effort.
In our analysis, using the logistic regression models, the power of the logistic regression tests of the $\beta_{2}$ parameter in the $\mathrm{F}_{2}$ and the $\beta_{1}$ parameter in the backcross was not monotonic in all cases. In fact, the power was low precisely when evidence for linkage was the strongest, namely when $r_{M Q}$ was small and the difference $\left(p_{2}-p_{1}\right)$ was large. This failure occurred due to a quasi-separation of points in the logit function. When $r_{M Q}$ was small and the difference $\left(p_{2}-p_{1}\right)$ was large, there are very few individuals with discordant marker trait pairs, resulting in difficulty fitting the logit function. The test statistic in the logistic regression is based upon a ratio of means, while the test statistic in the linear model is based upon a difference of means. Thus, when there are few 
Table 11. Comparison of estimates of $r_{M Q}$ and $p_{3}$ between the method of McIntyre et al. and interval regression $\left(n=100, r_{N Q}=0 \cdot 10\right)$

\begin{tabular}{|c|c|c|c|c|c|c|c|c|}
\hline \multirow[b]{3}{*}{$p_{1}$} & \multirow[b]{3}{*}{$p_{3}$} & \multirow[b]{3}{*}{$r_{M Q}$} & \multicolumn{4}{|c|}{ McIntyre et al.'s method } & \multirow{2}{*}{\multicolumn{2}{|c|}{$\begin{array}{l}\text { Interval regression } \\
\text { Two marker }\end{array}$}} \\
\hline & & & \multicolumn{2}{|l|}{ Single marker } & \multicolumn{2}{|l|}{ Two marker } & & \\
\hline & & & $\hat{r}_{M Q}\left(\operatorname{se}\left(\hat{r}_{M Q}\right)\right)$ & $\hat{p}_{3}\left(\operatorname{se}\left(\hat{p}_{3}\right)\right)$ & $\hat{r}_{M Q}\left(\operatorname{se}\left(\hat{r}_{M Q}\right)\right)$ & $\hat{p}_{3}\left(\operatorname{se}\left(\hat{p}_{3}\right)\right)$ & $\hat{r}_{M Q}\left(\operatorname{se}\left(\hat{r}_{M Q}\right)\right)$ & $\hat{p}_{3}\left(\operatorname{se}\left(\hat{p}_{3}\right)\right)$ \\
\hline \multirow[t]{4}{*}{$0 \cdot 00$} & $1 \cdot 00$ & $0 \cdot 20$ & $0 \cdot 197(0 \cdot 00148)$ & $1 \cdot 002(0.0026)$ & $0 \cdot 197(0 \cdot 001374)$ & $0.999(0.0018)$ & $0 \cdot 198(0 \cdot 0013)$ & $1 \cdot 002(0 \cdot 0015)$ \\
\hline & & $0 \cdot 30$ & $0 \cdot 298(0.00162)$ & $0.999(0.0029)$ & $0.299(0.001536)$ & $0 \cdot 9987(0$ & $0 \cdot 301(0 \cdot 0016)$ & $1 \cdot 010(0.0035)$ \\
\hline & & $0 \cdot 40$ & $0.402(0.00162)$ & $0.996(0.0031)$ & $0 \cdot 403(0 \cdot 001585)$ & $0.9993(0.0019)$ & $0.402(0.00158)$ & $1 \cdot 051(0.0077)$ \\
\hline & & $0 \cdot 50$ & $0.501(0.00162)$ & $0.997(0.0032)$ & $0 \cdot 501(0 \cdot 001581)$ & $0.9989(0.0020)$ & $0.470(0.00085)$ & $1 \cdot 035(0 \cdot 0100)$ \\
\hline \multirow{4}{*}{$0 \cdot 20$} & $0 \cdot 80$ & $0 \cdot 20$ & $0 \cdot 194(0.00306)$ & $0.800(0.0030)$ & $0.195(0.003005)$ & $0.8000(0.0029)$ & $0 \cdot 202(0.00162)$ & $0.801(0.0022)$ \\
\hline & & $0 \cdot 30$ & $0.291(0.00299)$ & $0.799(0.0030)$ & $0.293(0.002871)$ & $0 \cdot 7994(0 \cdot 0027)$ & $0 \cdot 297(0 \cdot 00179)$ & $0.807(0.0026)$ \\
\hline & & $0 \cdot 40$ & $0.401(0.00271)$ & $0.806(0.0032)$ & $0.401(0.002687)$ & $0.8035(0.0027)$ & $0.396(0.00171)$ & $0.843(0.0050)$ \\
\hline & & $0 \cdot 50$ & $0.499(0.00271)$ & $0.803(0.0032)$ & $0 \cdot 499(0 \cdot 0027)$ & $0 \cdot 8012(0 \cdot 0027)$ & $0 \cdot 464(0.001)$ & $0.908(0.0050)$ \\
\hline
\end{tabular}
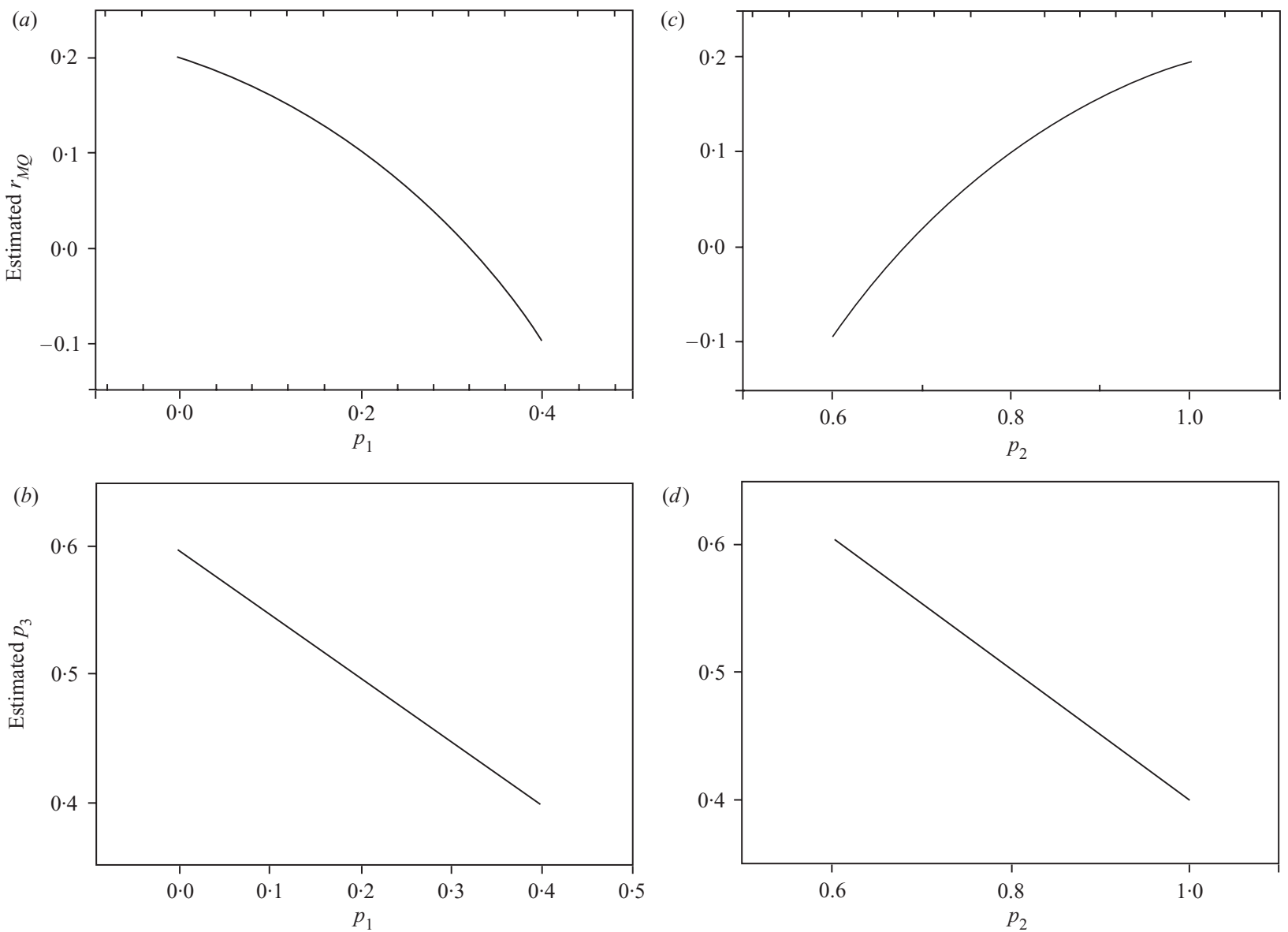

Fig. 5. (a) The effect of misspecification of $p_{1}$ on the estimation of recombination. (b) The effect of misspecification of $p_{1}$ on the estimation of $p_{3}$. (c) The effect of misspecification of $p_{2}$ on the estimation of recombination. $(d)$ The effect of misspecification of $p_{2}$ on the estimation of $p_{3}$.

discordant marker trait pairs in the linear model the difference between the marker means is maximized and the power of the test in the linear regression is correspondingly high. In our simulations, we found no cases where the power of the logistic model was better than that of the linear model. In fact, the power of the tests using the logistic model was no better and may be worse than the tests in the linear model. On 
the basis of these results, our recommendation is to use the linear model for detection of BTL with an appropriate corresponding permutation algorithm to determine the $P$ value empirically.

The estimators we present are moments estimators and are unbiased. However, they share a limitation inherent in all moment estimators, as they are not restricted to the parameter space in that the estimation of the penetrance $p_{3}$ is not automatically $0 \leqslant p_{3} \leqslant 1$, or $\hat{r}_{M Q}$ is not bounded in the interval $0 \leqslant \hat{r}_{M Q} \leqslant 0.50$. We do not present the joint estimation of all the $p_{i}$, instead we assume that $p_{1}$ and $p_{2}$ are known. We have shown that misspecification of $p_{1}$ and $p_{2}$ does lead to biased results, but that the impact is not large when misspecification is less than $20 \%$.

Our parameterization requires much less computational effort than previously published methods (Martinez \& Curnow, 1992 ; Haley \& Knott, 1992) as no interval stepping is required. The estimates of location $r_{M Q}$ are as accurate as the interval estimates and we can use a single marker for estimates of location, with no loss in accuracy (Table 11). Power is not directly comparable between our methods and other published methods (Martinez \& Curnow, 1992; Haley \& Knott, 1992) since the null hypotheses for the test statistics are different.

Based upon our parameterization, estimation of underlying threshold distributions is not needed, nor is any advance knowledge of the true genetic model. An experiment based upon two inbred lines, known to be different, can be conducted without the need for extensive testing of the $F_{1}$ phenotype. The $F_{1}$ phenotype or penetrance $\left(p_{3}\right)$ can be estimated from the backcross or $F_{2}$ progeny even without precise knowledge of the penetrance of the parental lines. Incorporating the estimation of the penetrance in the $F_{1}$ expands the options available to the experimenter, allowing for the dual purpose of detecting BTL and identifying the genetic model in a single set of measurements.

The method described is for a single gene and a binary trait. The model parameterization applies directly to categorical traits if the categorical trait is modelled in pairs of outcomes. To expand the parameterization to multiple genes requires a formulation of the joint distribution of $(Y, G, M)$ that includes multiple genes. Once the joint distribution has been expanded the expected values of the regression coefficients can be determined and the additional parameters estimated. It is important to note that in this approach every additional gene locus modelled requires an additional marker locus. This ensures that the model will be identifiable. That is, estimates for the additional recombination and penetrance parameters will be possible. If the model is expanded in this way, then the results of the current work should generalize to multiple genes.

\section{Appendix}

The expected log odds equations for the logistic model for the $\mathrm{F}_{2}$ experimental design

$$
\begin{aligned}
& e^{\beta_{0}}= \\
& {\left[\frac{p_{1}-2 p_{1} r_{M Q}+r_{M Q}^{2} p_{1}+2 r_{M Q} p_{3}-2 r_{M Q}^{2} p_{3}+p_{2} r_{M Q}^{2}}{1-\left(p_{1}-2 p_{1} r_{M Q}+r_{M Q}^{2} p_{1}+2 r_{M Q} p_{3}-2 r_{M Q}^{2} p_{3}+p_{2} r_{M Q}^{2}\right.}\right]} \\
& e^{\beta_{1}}= \\
& e^{\beta_{0}}\left[\frac{p_{1} r_{M Q}-p_{1} r_{M Q}^{2}+p_{3}-2 r_{M Q} p_{3}+2 r_{M Q}^{2} p_{3}+p_{2} r_{M Q}-p_{2} r_{M Q}^{2}}{1-\left(p_{1} r_{M Q}-p_{1} r_{M Q}^{2}+p_{3}-2 r_{M Q} p_{3}+2 r_{M Q}^{2} p_{3}+p_{2} r_{M Q}-p_{2} r_{M Q}^{2}\right)}\right] \\
& e^{\beta_{2}}= \\
& e^{\beta_{0}}\left[\frac{r_{M Q}^{2} p_{1}+2 r_{M Q} p_{3}-2 r_{M Q}^{2} p_{3}+p_{2}-2 p_{2} r_{M Q}+p_{2} r_{M Q}^{2}}{1-\left(r_{M Q}^{2} p_{1}+2 r_{M Q} p_{3}-2 r_{M Q}^{2} p_{3}+p_{2}-2 p_{2} r_{M Q}+p_{2} r_{M Q}^{2}\right)}\right] .
\end{aligned}
$$

This work is supported by NSF grant DBI 98-08026/0096044 (L.M.M., C.J.C., R.W.D.), NIH grant NIAAG16996(L. M. M.), USDA grant 98-35300-6173 (R.W.D.) and a Veterans Affairs Health Services Research Postdoctoral Fellowship (C.J.C.). The authors would like to thank Katy Simonsen, Assistant Professor of Statistics at Purdue University, Marie Davidian, Professor of Statistics North Carolina State University, and James Holland, USDA-ARS Research Geneticist, Department of Crop Science, North Carolina State University.

\section{References}

Agresti, A. (1990). Categorical Data Analysis. New York: Wiley.

Beckmann, J. \& Soller, M. (1988). Detection of linkage between marker loci and loci affecting quantitative traits in crosses between segregating populations. Theoretical and Applied Genetics 76, 228-236.

Churchill, G. \& Doerge, R. (1994). Empirical threshold values for quantitative trait mapping. Genetics 138, 963-971.

Doerge, R. \& Churchill, G. (1996). Permutation tests for multiple loci affecting a quantitative character. Genetics 142, 285-294.

Doerge, R. W., Zeng, Z.-B. \& Weir, B. S. (1997). Statistical issues in the search for genes affecting quantitative traits in experimental populations. Statistical Science 12, 195-219.

Elston, R. (1998). Methods of linkage analysis-and assumptions underlying them. American Journal of Human Genetics 63, 931-934.

Fain, P. (1978). Characteristics of simple sibship variance tests for the detection of major loci and application to height, weight and spatial performance. Annals of Human Genetics 42, 109-120.

Hackett, C. \& Weller, J. (1995). Genetic mapping of quantitative trait loci for traits with ordinal distributions. Biometrics 51, 1252-1263.

Haley, C. \& Knott, S. (1992). A simple regression method for mapping quantitative trait loci in crosses using flanking markers. Heredity 69, 315-324.

Hammond, K. \& James, J. (1970). Genes of large effect and the shape of the distribution of a quantitative character. Australian Journal of Biological Sciences 23, 867-876. 
Hosmer, D. \& Lemeshow, S. (1989). Applied Logistic Regression. New York: Wiley.

Jansen, R. (1992). A general mixture model for mapping quantitative trait loci by using molecular markers. Theoretical and Applied Genetics 85, 252-260.

Jansen, R. (1993). Interval mapping of multiple quantitative trait loci. Genetics 135, 205-211.

Kao, C. (1995). Statistical methods for locating the positions and analyzing epistasis of multiple quantitative trait genes using molecular marker information. PhD thesis, North Carolina State University.

Kruglyak, L. \& Lander, E. (1995). A nonparametric approach for mapping quantitative trait loci. Genetics 139, 1421-1428.

Luo, Z. \& Kearsey, M. (1989). Maximum likelihood estimation of linkage between a marker gene and a quantitative trait locus. Heredity 63, 401-408.

Luo, Z. \& Woolliams, J. (1993). Estimation of genetic parameters using linkage between a marker gene and a locus underlying a quantitative character in $\mathrm{F}_{2}$ populations. Heredity 70, 245-253.

Lynch, M. \& Walsh, B. (1998). Genetics and Analysis of Quantitative Traits. Sunderland, MA: Sinauer Associates.

Martinez, O. \& Curnow, R. (1992). Estimating the locations and the sizes of the effects of quantitative trait loci using flanking markers. Theoretical Applied Genetics 85, 480-488.

Mérat, P. (1968). Distributions de fréquences, interpretation du déterminisme génétique des caractères quantitatifs et recherche de 'genes majeurs'. Biometrics 24, 277-293.

O'Donald, P. (1971). The distribution of genotypes produced by alleles segregating at a number of loci. Heredity 26, 233-241.
Olson, J., Witte, J. \& Elston, R. (1999). Tutorial in biostatistics genetic mapping of complex traits. Statistics in Medicine 18, 2961-2981.

Paterson, A. (1998). Molecular Dissection of Complex Traits. Boca Raton, FL: CRC Press.

Rebaï, A. (1997). Comparison of methods for regression interval mapping in QTL analysis with non-normal traits. Genetical Research 69, 69-74.

Searle, S. R. (1997). Linear Models. New York: Wiley.

Thompson, E. (1998). Inferring gene ancestry: estimating gene descent. International Statistical Review 66, 29-40.

Visscher, P., Haley, C. \& Knott, S. (1996). Mapping QTLs for binary traits in backross and $\mathrm{F}_{2}$ populations. Genetical Research 68, 55-63.

Weller, J. (1986). Maximum likelihood techniques for the mapping and analysis of quantitative trait loci with the aid of genetic markers. Biometrics 42, 627-640.

Wilcox, P. (1995). Genetic dissection of fusiform rust resistance in loblolly pine. $\mathrm{PhD}$ thesis, North Carolina State University

Wright, S. (1952). The genetics of quantitative variability. In Bull. Agricultural Research Council: Quantitative Inheritance, pp. 5-41. London: Her Majesty's Stationery Office.

$\mathrm{Xu}, \mathrm{S}$. (1996). Computation of the full likelihood function for estimating variance at a quantitative trait locus. Genetics 144, 1951-1960.

Xu, S. \& Atchley, W. (1996). Mapping quantitative trait loci for complex binary diseases using line crosses. Genetics 143, 1417-1424.

Zeng, Z.-B. (1993). Theoretical basis of precision mapping of quantitative trait loci. Proceedings of the National Academy of Sciences of the USA 90, 10972-10976.

Zeng, Z.-B. (1994). Precision mapping of quantitative trait loci. Genetics 136, 1457-1468. 